\title{
Artículo de Revisión/ Review Article \\ Teorías y modelos aplicados al estudio de la conducta del \\ pescador: implicaciones en procesos de gestión
}

\section{Theories and models used in analyzing fishermen behavior: insights on fisheries management processes}

\author{
Helven Naranjo-Madrigal ${ }^{1}, 2 *$
}

\begin{abstract}
RESUMEN
En el proceso de toma de decisiones para el manejo de pesquerías se ha incrementado la atención en considerar aspectos de la dimensión humana de la pesca adicionalmente a la dimensión ecológica. En la dimensión humana, el componente de dinámica de flotas pesqueras se ha analizado a través de modelos y teorías que han dado más énfasis al comportamiento de embarcaciones y menor atención al comportamiento del pescador como individuo o grupos de individuos. La presente revisión de literatura se enfoca en estudios que han analizado aspectos de la conducta del pescador mediante teorías y modelos que logran explicar y predecir su comportamiento. Se encontró que los motivadores económicos per se no logran explicar la complejidad del proceso de toma de decisiones del pescador, más bien una gran cantidad de aspectos no monetarios están implícitos en las elecciones que hacen los pescadores en el corto y largo plazos. Modelos que incorporan información de distintas disciplinas y enfoques transdisciplinarios logran explicar mejor las decisiones del pescador asociadas al esfuerzo, incentivos económicos, capturas, regulaciones, aspectos de su cultura, forma de vida, estrategias de sobrevivencia, satisfacción laboral y percepción del bienestar. A partir de esta revisión de literatura se propone un marco conceptual de manejo socioecológico para integrar las motivaciones de la conducta de los pescadores y la relación con los ecosistemas de los cuales estos dependen.
\end{abstract}

Palabras claves: Conducta del pescador, gobernanza, manejo, modelos, sistemas socioecológicos.

\begin{abstract}
As part of the decision-making process in fisheries management, attention to aspects with regard to regarding the human dimension of fishing has increased, in addition to the ecological dimension. Within the human dimension, the fishing fleet dynamics component has been analyzed using models and theories that emphasize fleet behavior and pay less attention to fishermen's behavior as an individual or groups of individuals. This literature review is focused on studies that have analyzed aspects of fishermen behavior using theories and models that explain and predict their behavior. It was found that economic drivers per se do not explain the complexity of the fisherman's decision-making process; on the contrary, a number of non-monetary aspects are implicit in the choices made by fishermen in the short and long term. Models that include information from different disciplines and trans-disciplinary approaches better explain fishermen's decisions in relation to effort, economic incentives, catches, regulations, and aspects related to their culture, lifestyle, livelihood strategies, job satisfaction and perception of wellbeing. Based on this literature review, a conceptual framework is proposed for socio-ecological management in order to integrate the drivers related to fishermen behavior and their relationship with the ecosystems on which they depend.
\end{abstract}

Keywords: Fishermen's behavior, governance, management, models, socio-ecological systems.

\footnotetext{
Consultant services department, ALNA S.A., PO Box 11401, San José, Costa Rica

2 Programa de Maestría en Ciencias Marinas y Costeras. Universidad Nacional, Apdo. 86-3000, Heredia, Costa Rica. helvenn@hotmail.com
}

Recibido: 25 de abril de 2016

Corregido: 13 de octubre de 2016

Aceptado: 25 de octubre de 2016

DOI: http://dx.doi.org/10.15359/revmar.9-1.4 


\section{INTRODUCCIÓN}

Los seres humanos han aprovechado las poblaciones marinas para la alimentación, el beneficio económico y tradiciones culturales por miles de años (Plagányi et al. 2013). Según Pitcher y Cheung (2013) en los últimos 150 años se ha visto un enorme aumento sin precedentes en el alcance geográfico y el rango de profundidad de la intensidad de pesca a través de nuevas tecnologías y una mercantilización global de los productos de la pesca. Además, la FAO (2012) advierte que las capturas de la pesca marina no han aumentado significativamente desde 1980 y existe evidencia de episodios de agotamiento según la ubicación y la profundidad (por ejemplo, Pitcher \& Cheung, 2013).

Algunos autores señalan que existe una recuperación importante en aquellos stocks que han sido evaluados y manejados eficientemente y que la recuperación de poblaciones sobreexplotadas es factible siempre y cuando exista una adecuada gobernanza (Hilborn, 2007). Sin embargo, otros autores mencionan (Worm \& Branch, 2012; Pitcher \& Cheung, 2013) que debido a que la mayor parte de las capturas en el mundo y la alta biodiversidad se concentran en "regiones calientes" (Worm \& Branch, 2012), con escasas capacidades de evaluación y manejo (por ejemplo, países subdesarrollados donde predominan pesquerías de pequeña escala), la situación aún hoy es crítica. Como resultado de este debate, se ha expresado la urgencia de implementar sistemas de manejo integrales que incorporen el comanejo, derechos de propiedad local, áreas marinas protegidas, entre otras herramientas que permitan alcanzar la sostenibilidad en el uso de los recursos y la resiliencia socioecológica (Pomeroy \& Berkes, 1997; Österblom et al. 2013).

Independientemente del sistema de manejo utilizado para abordar la problemática de las pesquerías, es evidente la necesidad de dar un mayor énfasis a la dimensión humana en su manejo, la cual es una fuente de alta incertidumbre en los procesos de manejo (Fulton et al. 2011) y abarca aspectos de la dinámica de flotas, políticos, institucionales, dinámica de mercados y factores socioculturales propios de las comunidades pesqueras (van Putten et al. 2012; Österblom et al. 2013).

El objetivo del presente documento fue realizar una revisión de las teorías y los modelos que se han utilizado para estudiar la conducta del pescador y sus implicaciones en los procesos de gestión pesquera. Se discute sobre la relevancia y las limitaciones de los modelos y las teorías en capturar las motivaciones detrás de la conducta del pescador. A partir de la revisión bibliográfica se exploran las variables incluidas en cada una de las publicaciones que analizan la conducta del pescador con el fin de proponer un modelo conceptual de manejo robusto e integral, que sirva como una hoja de ruta para el desarrollo de líneas de investigación y construcción de modelos socioecológicos que auxilien la incorporación de este conocimiento en los procesos de gestión de pesquerías. 


\section{MATERIALES Y MÉTODOS}

Laliteraturarevisadaenesteanálisis incluyó la búsqueda de documentos en motores de base de datos como Web of Knowlegde, ScienceDirect, Web of Science, entre otras. Los criterios de búsqueda incluyeron palabras claves como: conducta humana, conducta del pescador, asignación del esfuerzo, toma de decisiones, antropología de la pesca, teoría del forrajeo, maximización del beneficio, teoría del juego, modelos de elección discreta, modelos bayesianos, redes sociales y dinámica de flotas pesqueras. Los resultados fueron filtrados y se retuvieron aquellas publicaciones relacionadas con el tema de la pesca y el manejo de recursos naturales.

En esta revisión se consideraron aquellas publicaciones que lograban analizar aspectos de la conducta de pescadores, ya que los estudios que analizan el comportamiento de flotas y/o embarcaciones limitan capturar la complejidad del comportamiento del pescador (Béné \& Tewfik, 2001). Aunque ciertas teorías y modelos se han aplicado en ambos casos.

Se categorizaron las variables utilizadas para estudiar la conducta del pescador en cada caso de estudio, con respecto al ámbito al que pertenecían como: sociales (por ejemplo, etnográficas), económicas, sicológicas (por ejemplo, cognitivas), culturales (por ejemplo, tradiciones), tecnológicas, ambientales y del recurso. Para cada publicación revisada, se registró la presencia o no de cada una de las variables incluidas en el proceso de modelación y/o análisis teóricoconceptual. Esto permitió generar una base de datos que ayudó a facilitar el análisis de la literatura consultada.

A partir del análisis exploratorio y de una revisión crítica de la literatura asociada al tema, se construyó un modelo conceptual de manejo que sintetiza el conocimiento inter y transdisciplinario de los distintos enfoques teóricos y análisis de casos de estudio. Para la construcción del modelo conceptual se utilizaron las herramientas de formas y conectores de diagramación en el programa Power Point 2013.

\section{RESULTADOS}

\section{Revisión sistemática de aproximacio- nes teóricas y modelación analítica}

La figura 1 muestra la cantidad de veces que variables de distinta índole fueron incluidas en publicaciones que abordaron el estudio de la conducta del pescador a través de diferentes aproximaciones teóricas y modelaciones analíticas. Se observa la predominancia de motivadores económicos (27\%) que han sido parte del análisis de la conducta del pescador según la teoría de maximización del beneficio (42.1\%) en diversos casos de estudio y en menor grado con el uso de modelos de elección discreta $(30.7 \%)$ y otros enfoques. La constante presencia de variables económicas es la que provoca mayor asimetría con respecto al uso de otras variables de aproximaciones teóricas y modelaciones analíticas.

Los modelos que lograron incorporar mayor variedad de factores 


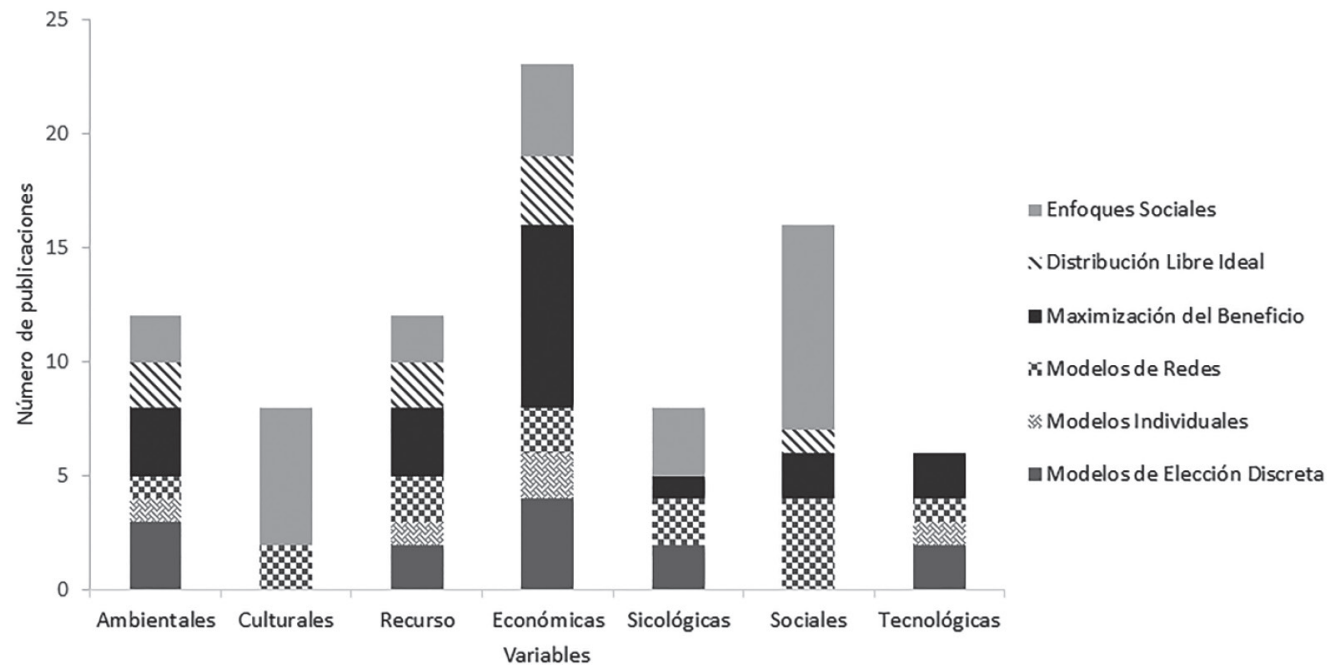

Fig. 1. Categorización de variables incluidas en distintas aproximaciones teóricas y de modelación que estudian la conducta del pescador. Resultado de 42 publicaciones revisadas Fig. 1. Categorization of variables included within modeling and theorical approaches. It shows the results of 42 publications reviewed

de distinta índole fueron los modelos de redes. Por otra parte, las variables con menor presencia en estudios de la conducta del pescador fueron las variables asociadas a aspectos culturales (9.4\%) dada su naturaleza cualitativa.

Aspectos conceptuales de la conducta humana y conducta del pescador

La conducta humana es definida como el conjunto de comportamientos exhibidos por los seres humanos, influenciados por la cultura, el medio ambiente, las actitudes, las emociones, los valores, la ética, la autoridad, la relación, la hipnosis, la persuasión, la coacción y/o la genética (Anderson \& Carter, 1990). Esta definición revela la gran complejidad que existe al tratar de analizar y entender la conducta humana y esta tarea ha sido una parte importante de la sicología cognitiva. Según esta ciencia, existen múltiples factores que influyen en la toma de decisiones de los seres humanos como: la experiencia pasada, los sesgos cognitivos, la edad y las diferencias individuales, la creencia en la relevancia personal y una escalada de compromiso (Stanovich \& West, 2008). La comprensión de estos factores en la toma de decisiones es un proceso importante para entender cuáles decisiones son tomadas. Es decir, los factores que influyen en el proceso pueden afectar los resultados (Dietrich, 2010).

En el contexto de la pesca, la importancia de entender las decisiones que realizan los pescadores es poder predecir un comportamiento racional, lo cual tiene implicaciones en el manejo. Sin embargo, como se observó en la figura 1, la mayoría de los estudios asociados al comportamiento de los pescadores se han enfocado según 
el prisma de la economía, que considera acciones racionales aquellas que buscan la utilidad, la optimización de la riqueza, la búsqueda de rentas, la minimización de costos, el cálculo de costo-beneficio y similares (Mas-Colell et al. 1995). A partir de este concepto económico de racionalidad, Gordon (1953) propuso un modelo bioeconómico, con el cual asumió que en pescadores promedio con conducta homogénea, la predicción de su distribución del esfuerzo es simple, porque está determinada por los rendimientos económicos esperados de pescadores individuales y en sitios y pesquerías alternativas. No obstante, estudios empíricos revelan que los modelos simples, que se basan en supuestos de racionalidad económica al analizar el esfuerzo, no proporcionan predicciones exactas de las distribuciones del esfuerzo (Béné \& Tewfik, 2001). Incluso en una pesquería monoespecífica, donde el proceso de toma de decisiones en la asignación de esfuerzo espacio-temporal se aplica a una especie, la distribución del esfuerzo es el resultado de elecciones en la que influyen aspectos como: los patrones tradicionales del esfuerzo de los pescadores (información pasada), aversión o no aversión al riesgo, expectativas de captura y costos, tecnología disponible, regulaciones de manejo, disponibilidad del recurso y condiciones de mercado (Béné \& Tewfik, 2001; Salas et al. 2004).

Recientemente, y en respuesta a solventar los fracasos en el manejo pesquero debido a la incertidumbre proveniente de la dinámica del pescador y su interacción con el entorno, se ha optado por utilizar una definición de "racional" más amplia, que la describe como "comprensible", una vez que se considera un conjunto suficiente de "motivaciones", teniendo en cuenta que estas motivaciones son propensas a extenderse más allá de la economía reduccionista y serán distintas en diferentes culturas y entre los sistemas con diversidad social, económica y entornos geográficos (Fulton et al. 2011). Según esta definición se discutirán los alcances de las publicaciones que han analizado la conducta del pescador y que han sido parte de la revisión en este estudio.

Hay distintas escalas que se consideran cuando se estudia el proceso de toma de decisiones de los pescadores. Desde el punto de vista del esfuerzo pesquero, las decisiones que toman los pescadores han sido categorizadas según conceptos militares (Handel, 1989), aquellas que se extienden en el corto plazo representadas por acciones individuales designadas a alcanzar un objetivo inmediato se les llama "tácticas" (por ejemplo, movimientos de embarcaciones en zonas de pesca siguiendo movimientos de cardúmenes en un viaje de pesca) y aquellas decisiones que se extienden en el largo plazo se les conoce como "estrategias" (por ejemplo, decisión de cambiar la especie objetivo en una temporada de pesca para aumentar el ingreso) (Salas \& Gaertner, 2004). Aunque científicos pesqueros han tratado de cuantificar el proceso de 
toma de decisiones y las elecciones asociadas procurando captar incentivos monetarios y no monetarios, existen aspectos complejos de la conducta del pescador que generan incertidumbre, lo que tiene repercusiones en el manejo. Según Béné \& Tewfik (2001), es imprescindible utilizar enfoques multicriterio y sistémicos para abordar la complejidad de la conducta del pescador. van Putten et al. (2012) en una revisión de publicaciones sobre dinámica de flotas encontraron que la mayoría se enfocaron en pesquerías industriales comerciales y aunque una larga proporción de estos estudios consideraron características individuales (por ejemplo, características de las embarcaciones) como motivadores claves de las elecciones, las motivaciones económicas per se dominaron. Lo anterior es consistente al revisar las publicaciones de la conducta del pescador, en la figura 1 se observa una mayor cantidad de estudios que incluyeron motivaciones económicas.

\section{Modelos aplicados al estudio de la conducta del pescador \\ Modelos de Elección Discreta}

Los modelos de elección discreta pretenden representar el mecanismo de elección de un individuo que se enfrenta a una serie de alternativas entre las que únicamente puede escoger una de ellas. Estos modelos están basados en el principio de la utilidad aleatoria en la que cada individuo $n$ tiene una función de utilidad Uin asociada a cada una de las alternativas $i$, escogiendo aquella alternativa que maximiza su utilidad.
Esta función de utilidad requiere de dos componentes (Fukuda \& Yai, 2010): i) un componente determinístico o sistemático que puede ser explicado por factores observables o variables explicativas; ii) un componente aleatorio que exhibe variaciones no observadas entre individuos como errores de medición o diferencias entre individuos. La flexibilidad de estos modelos radica en la posibilidad de especificar cualquier distribución en la estructura del error, relaciones lineales paramétricas y relaciones no lineales.

En estos modelos se han incorporado aspectos de elección, tanto monetarios como no monetarios, en los que se incluyen actitudes ante el riesgo, aspectos ambientales y del recurso. El uso de estos modelos en el análisis de la conducta del pescador ha estado restringido a estudios que analizan las decisiones de los pescadores en la elección de especies objetivo y sitios de pesca, con gran énfasis en los supuestos de la teoría de maximización del beneficio (Salas et al. 2004). Por ejemplo, Holland \& Sutinen (1999) utilizaron datos de una pesquería de arrastre para analizar, mediante un modelo de elección discreta, las decisiones en la distribución del esfuerzo de pescadores en Nueva Inglaterra. Su propuesta para construir estos modelos fue elegir variables que afectaban los beneficios esperados como: información del promedio de ingreso enáreas visitadas con anterioridad, incorporación de la condición de aversión al riesgo utilizando como variable proxy el coeficiente de variación de ingresos (con el supuesto de que pescadores con aversión al riesgo 
preferían sitios con bajo coeficiente de variación de ingresos) y tiempo de viaje a los sitios de pesca. El modelo permitió predecir el nivel del esfuerzo de embarcaciones en distintas áreas, y con ciertas condiciones regulatorias, con importantes implicaciones en las políticas de manejo. Aunque los autores aseguraron haber recolectado información etnográfica a partir de entrevistas, no incluyeron informaciones como experiencia de pesca, posición familiar o edad de los tripulantes dentro del modelo, las cuales pueden tener peso en las decisiones de pesca.

Uno de los estudios que aborda la conducta del pescador fue realizado por Salas et al. (2004). Estos autores evaluaron las decisiones de los pescadores artesanales de pulpo y langosta en tres comunidades en Yucatán, mediante modelos de elección discreta. El supuesto en la construcción de estos modelos fue asumir que los pescadores eran "agentes económicos", que tenían como objetivo obtener los mayores ingresos al elegir trabajar en la pesca de la especie de mayor valor económico (langosta). También, los modelos permitieron explorar cambios en la disponibilidad de recursos y selección aleatoria. Los resultados mostraron que los pescadores no operaban al azar, ya que consideraban la información sobre los recursos disponibles e ingresos generados en viajes anteriores, antes de seleccionar o cambiar la especie objetivo. Además, estos autores confirmaron que la teoría económica explicó parcialmente los resultados encontrados debido a incentivos no monetarios como la habilidad de los pescadores.

\section{Modelos Individuales}

Los modelos individuales tratan de simular las decisiones de pescadores individuales mediante una serie de variables que varían según los individuos. En una definición más técnica formulada por Railsback (2001): "Son modelos que describen a los individuos o partes que configuran un sistema como entidades autónomas y discretas, y focalizan toda su atención en caracterizar estas partes discretas mediante reglas de comportamiento, permitiendo que estas partes interaccionen entre ellas y con el entorno en el que se hallan". El uso de estos modelos en el análisis de la conducta individual de pescadores ha sido muy limitado, solo el $5.8 \%$ de los artículos revisados utilizaron estos modelos. Aunque se pueden encontrar numerosos estudios en conducta de flotas. Por ejemplo, Dreyfus-León (1999) generó un pescador artificial el cual llamó "fishermat", con el propósito de analizar sus estrategias en la búsqueda de la captura mediante un proceso de aprendizaje utilizando redes artificiales neuronales. La información virtual incorporada tomó en cuenta la distancia de las áreas de pesca al puerto pesquero y los sitios de mayor densidad de peces.

La ventaja de estos modelos reside en su capacidad de procesamiento, ya que es posible incorporar un sinnúmero de variables de distinta índole (económicas, ambientales y sociales) que pueden influir en las decisiones ante distintos escenarios, con diferentes propiedades ambientales y espacio-temporales. 


\section{Modelos de Redes}

Los modelos de redes son herramientas interdisciplinarias de gran auge en el estudio de sistemas socioecológicos, las cuales se centran en la representación, análisis y modelado de los sistemas sociales, biológicos y tecnológicos complejos en forma de redes gráficas. La capacidad de estas herramientas en incorporar múltiples interconexiones como ecosistemas, redes tróficas biológicas y otros procesos de la dimensión humana como redes institucionales e información socioeconómica permite el análisis de diversas representaciones del mundo real a diferentes escalas (Kolaczyk \& Csárdi, 2014).

La capacidad de estos modelos en combinar variables cualitativas y cuantitativas explica el porqué los estudios de caso analizados en la revisión de literatura, registraron mayor diversidad de variables con el uso de este tipo de modelos. Estos modelos han sido aplicados en el análisis de las relaciones que establecen los pescadores al compartir información sobre tendencias de capturas, uso de tecnología asociada a las operaciones de pesca, aspectos del mercado, estrategias de pesca, cumplimiento de regulaciones y factores climáticos (Naranjo-Madrigal et al. 2015; Stevens et al. 2015). Se destacan los Modelos de Redes Sociales (MRSs) y los Modelos de Redes Bayesianas (MRBs) en el estudio de la conducta del pescador.

Las propiedades de las redes están definidas por su capacidad para realizar análisis descriptivos y numéricos a través de gráficos de redes, así como las características de la estructura de la red como: centralidad, densidad, transitividad, agrupamiento y modularidad (Kolaczyk \& Csárdi, 2014). Estas características han sido relacionadas con el estudio de la dinámica de redes sociales y aspectos del manejo adaptativo como: confianza, memoria social, redundancia, heterogeneidad, aprendizaje y capacidad adaptativa.

Los MRSs en el contexto de la pesca se han enfocado en el estudio de factores que transcienden las motivaciones económicas. Por ejemplo, en la investigación realizada por Stevens et al. (2015) se buscó entender las motivaciones que hacen que los pescadores de pequeña escala vigilen el cumplimiento de regulaciones en sus sitios de pesca y recursos en varias comunidades de la costa Atlántica de Nicaragua. En los modelos se incluyeron factores como: ingresos, seguridad alimentaria, edad y vigilancia comunitaria de los pescadores. Algunos de estos factores explicaron el nivel de compromiso y, por ende, vigilancia de los usuarios con respecto al acatamiento de regulaciones, las cuales estuvieron en función del nivel de acompañamiento institucional en la vigilancia y control de la pesca ilegal.

Por otra parte, los MRBs son herramientas estadísticas no paramétricas, que se basan en la inferencia bayesiana para deducir la influencia de variables explicativas sobre salidas de interés. El MRB es una representación en forma de red de interrelaciones y dependen- 
cias condicionales entre un conjunto de variables (Pearl, 1998). Los MRBs son especialmente adecuados para analizar las opciones en la toma de decisiones, ya que permiten la inclusión de causalidad y dependencias jerárquicas. Además, los MRBs son fáciles de actualizar cuando se obtiene nueva información y es posible calibrarlos y validarlos. Así, los modelos MRBs encajan bien con los conceptos de la gestión adaptativa y pueden ser una herramienta útil para organizar el conocimiento actual, la generación de hipótesis contrastables y la comparación de alternativas (Prato, 2005). Otra ventaja importante de los MRBs es su capacidad de incorporar el conocimiento de expertos y los datos empíricos en un mismo análisis. Esta flexibilidad permite introducir toma de decisiones subjetivas y razonamiento cualitativo.

A pesar de la gran capacidad que tienen los MRBs en analizar datos cuantitativos y cualitativos, su uso en el análisis de aspectos de la conducta del pescador ha sido limitado. van Putten et al. (2013) analizaron factores que influyen en la participación de pescadores en actividades pesqueras en Australia, considerando motivaciones económicas y socioculturales. Estos autores exploraron distintos escenarios que incorporaban variables como: costos de combustible, precio de las especies, disponibilidad de infraestructura, entre otras. A partir de los resultados de este estudio los autores derivaron aspectos importantes para mejorar la capacidad en el manejo de la pesquería.
En el estudio de Naranjo-Madrigal et al. (2015) sobre las decisiones que toman los pescadores de buceo artesanal en la elección de los sitios de pesca, mediante un MRB, se incorporó información empírica cuantitativa derivada de información de los viajes de pesca (captura, visibilidad, especies, etc.) y cualitativa recolectada a partir de encuestas, entrevistas y observación participativa con los pescadores (experiencia, posición familiar, etc.). Los MRBs permitieron obtener predicciones realísticas sobre los factores que influyeron en la elección de los sitios de pesca y con base en la información descriptiva de la observación participativa, percepción de pescadores y opinión de expertos, se establecieron relaciones causa-efecto que definieron la importancia de los factores ambientales en la asignación espacial del esfuerzo y la condición de no causalidad de variables económicas en las elecciones de los sitios de buceo.

\section{Teorías aplicadas al estudio de la conducta del pescador}

Teoría de Maximización del Beneficio

La teoría económica aplicada al estudio de pesquerías considera a los pescadores, las embarcaciones y flotas como productores o empresas, los cuales maximizan su beneficio económico (Cabrera \& Defeo, 2001), el cual es la diferencia entre los ingresos obtenidos por la venta de la captura menos los costos que se invirtieron al obtener la captura. Esta teoría de la microeconomía intenta explicar cómo se generan las decisiones que toman 
los pescadores basados en expectativas de los ingresos y costos asociados a elecciones alternativas de producción, las que pueden ser caracterizadas con distintos grados de incertidumbre (van Putten et al. 2012). Diversos estudios han utilizado esta teoría para modelar y predecir la conducta de flotas (Holland \& Sutinen, 1999; Cabrera \& Defeo, 2001) más que para predecir las decisiones que toman pescadores individuales (Salas et al. 2004). En estos últimos casos, se ha dado énfasis a supuestos asociados a la relación costo-beneficio, del recurso (CPUE) y ambientales (distancia al puerto, sitios de pesca) sin incorporar otras variables implícitas en la conducta del pescador (por ejemplo, socioculturales).

Por ejemplo, Holland (2008) en un estudio que comprendió una mezcla de conceptos de la sicología cognitiva, conducta económica y entrevistas etnográficas comprobó que existen factores (por ejemplo, aversión al riesgo) que promueven incertidumbre en las decisiones de pescadores como individuos económicamente racionales. Otros autores encontraron que factores como tradiciones familiares e inercia no son consistentes con el objetivo de maximización del beneficio (Bockstael \& Opaluch, 1983).

Dados los altos costos que incurre realizar una efectiva vigilancia de las regulaciones, existe un gran interés de las autoridades administrativas de los recursos pesqueros por mejorar el compromiso de los pescadores con los objetivos de manejo, para así lograr un mejor acatamiento de las medidas regulatorias. La teoría de maximización del beneficio ha sido utilizada para estudiar la conducta de acatamiento de estas regulaciones por parte de los pescadores. En este caso, los análisis se han basado en el enfoque de la disuasión y el enfoque normativo (Nøstbakken, 2008), los cuales se integran a elementos de la teoría neoclásica (por ejemplo, elección racional) y la ley criminal (por ejemplo, castigo). Estudios en este campo tratan de determinar la conducta ilegal de no acatamiento con aspectos como la recompensa (por ejemplo, valor marginal de producto ilegal) con respecto a niveles de castigo (por ejemplo, decomiso de equipo o multas) necesarios para disuadir la conducta ilegal (Al-Subhi et al. 2013). Se han utilizado modelos de elección y disuasión para explorar estos aspectos, proporcionando buenas predicciones (por ejemplo, Kuperan \& Sutinen, 1998).

Otros autores, relacionados con el tema de la sicología cognitiva y social, argumentan que es necesario incluir otros factores que tienen implicaciones importantes en las motivaciones de acatamiento como: moralidad social, normas sociales, percepción de justicia, comunicación con contraparte administrativa, confianza mutua y resolución de conflictos (May, 2004). Al-Subhi et al. (2013) señalan que la mayoría de las aproximaciones utilizadas para analizar la conducta de acatamiento trata a las regulaciones como directrices coercitivas en lugar de contratos sociales que ayudan a generar compromisos compartidos. 
Además, estos autores argumentan que la falta de consulta a los pescadores, fomentada por un sistema de manejo de "comando-control", lleva a ineficientes decisiones, lo que genera altos niveles de inconformidad entre los pescadores que afecta el acatamiento de las regulaciones. Por tal motivo, modelos híbridos de elección, que incorporan un amplio espectro de variables que influyen en las motivaciones de acatamiento en pesquerías de pequeña escala, han sido desarrollados recientemente (Al-Subhi et al. 2013).

La limitación que representa abordar la complejidad del proceso de toma de decisiones de pescadores de pequeña escala a través de la teoría de maximización del beneficio ha sido confirmada en estudios empíricos que han utilizado información más variada, que incorpora aspectos del pescador y ambientales propios del contexto en el cual se desarrolla la pesquería (Béné \& Tewfik, 2001; Stevenson et al. 2011). Por ejemplo, Béné \& Tewfik (2001), en una crítica a los análisis sobre la asignación del esfuerzo que utilizan datos agregados (información de una flota) según la premisa de la maximización del beneficio económico, advierten de dos errores: i) las interpretaciones que se basan en datos agregados están limitadas por la dificultad en capturar la lógica y el comportamiento que caracterizan a los pescadores como individuos; ii) el comportamiento individual (e incluso el colectivo) es el resultado de una combinación de factores y consideraciones que van más allá del sencillo principio de maximización de ganancias. Stevenson et al. (2011) analizaron la conducta de buzos comerciales y su efecto en la productividad en una pesquería de peces ornamentales y encontraron que aspectos como la cooperación, alta satisfacción laboral y otros incentivos no monetarios, son de importancia en el entendimiento de los patrones del esfuerzo de pesca.

\section{Teoría de la Distribución Libre Ideal}

La teoría de la distribución libre ideal pertenece al estudio de la ecología del comportamiento de forrajeadores (teoría del forrajeo) que predice la distribución de forrajeadores y el éxito de forrajeo entre áreas (Fretwell, 1972). Esta teoría explica la distribución animal en términos de decisiones individuales, describiendo la distribución en el sentido "ideal" al considerar que todos los individuos son "iguales" en las habilidades competitivas, y "libres" en el sentido de que son capaces de estar en cualquier parte sin restricciones en los costos. El uso de esta teoría se aplica más comúnmente en la perspectiva de la biología pesquera que en la economía pesquera. En la biología pesquera, los pescadores se ven como depredadores, mientras que, en la economía pesquera, son vistos como productores o empresas (van Putten et al. 2012).

En esta teoría, solo se registra un estudio que analiza las decisiones de pescadores como individuos más que como flotas. Abernethy et al. (2007) analizaron las decisiones en la elección de los sitios de pesca de 
pescadores de pequeña escala en la isla de Anguila a partir del supuesto de que los pescadores persiguen el objetivo de alcanzar los más altos premios posibles, por lo que su comportamiento está basado en la maximización del beneficio. Estos autores encontraron que el comportamiento de los pescadores no fue consistente con los supuestos de la teoría de la distribución libre ideal y dieron dos razones: i) la inexacta información de los pescadores en la disponibilidad del recurso hizo que estos no aumentaran su esfuerzo en aquellas áreas de mayor disponibilidad, en otras palabras, su distribución no fue "ideal"; ii) no todos los pescadores fueron "libres" para distribuirse entre los arrecifes debido a la variación en características sociales, económicas y físicas lo que limitaba sus movimientos $\mathrm{y}$, por ende, su capacidad de extraer recursos.

\section{Teoría Social: Enfoques en la Pesca}

Los científicos sociales han reconocido la importancia de abordar la evaluación y manejo de pesquerías no solo tomando en cuenta el componente biológico, sino también la dimensión humana. Sin embargo, estos advierten que este componente ha sido desequilibradamente desarrollado en términos económicos debido a que los pescadores son vistos como seres controlados por incentivos económicos $\mathrm{y}$ necesitan de una fuerte autoridad capaz de controlar sus actividades pesqueras según la legislación existente (Reed et al. 2013). Esta realidad se refleja en la gran cantidad de estudios sobre la conducta del pescador y dinámica de flotas y como veremos más adelante, este modelo ha predominado en esquemas de manejo.

En la teoría social, existen varios enfoques con los que se ha estudiado el proceso de toma de decisiones, actitudes y percepciones que influyen en la conducta de los pescadores $\mathrm{y}$ explican las motivaciones de su racionalidad. Algunos de estos enfoques se asocian al bienestar de los pescadores (Weeratunge et al. 2013), las estrategias de sobrevivencia (Busilacchi et al. 2013) que desarrollan a partir de su interacción con el entorno, el apego al trabajo y la satisfacción laboral (Pollnac \& Poggie, 2006) que representa la pesca como estilo de vida (Pollnac \& Poggie, 2008; Reed et al. 2013), además de conceptos como el Paisaje Cultural (Davidson-Hunt, 2003) y el conocimiento ecológico tradicional (Berkes, 1999) que tratan de explicar el conocimiento adquirido, tradiciones, valores y costumbres inherentes a su cultura. Debido al traslape de enfoques que existe en estudios sobre estos aspectos, en esta sección no se describen de manera separada.

Reed et al. (2013) en un estudio sobre el papel sociocultural de la pesca en varios puertos pesqueros en Inglaterra encontró que, si bien la pesca no se defiende como una parte vital de la economía local, la población rural la valora por su contribución social y cultural a la comunidad. Este resultado se debió a la opinión de que la pesca crea un sentido de pertenencia geográfica que se valora como parte de 
la identidad individual y comunitaria. Lo anterior reafirma lo encontrado en otros estudios (Ota \& Just, 2008) que demuestran que en este tipo de comunidades los grupos ocupacionales persiguen mantener su estilo de vida, éxito que no se define principalmente por éxito financiero. Este resultado es consistente con lo expuesto por Álvarez et al. (2012), quienes indican que las comunidades pesqueras $\mathrm{y}$ las conductas de los individuos que la forman, están definidas por la manera como estos desarrollan una identidad particular, que explica el uso de los recursos que dependen para sobrevivir. A esta relación DavidsonHunt (2003) la llamó Paisaje Cultural (PC), la cual es la expresión física del complejo y dinámico conjunto de relaciones, procesos y vínculos entre las sociedades y los entornos.

Aspectos como tradiciones, valores $\mathrm{y}$ percepciones presentes en comunidades pesqueras influyen en el comportamiento de los pescadores $\mathrm{y}$ estos saberes han sido estudiados según el concepto del Conocimiento Ecológico Tradicional. Este concepto, propuesto por Berkes (1999) y utilizado en análisis de comunidades pesqueras por otros autores (Butler et al. 2012), es caracterizado por aquellas habilidades prácticas y sabiduría desarrolladas por los pescadores en una escala local, a través de estrategias de subsistencia asociadas a su entorno a lo largo de generaciones sucesivas. En el mundo, se reconoce este conocimiento en la gestión contemporánea de los recursos naturales y es representado, por ejemplo, como el conocimiento local de los pescadores sobre las especies, sus historias de vida, distribuciones, etc. (Butler et al. 2012).

Este conocimiento tiene implicaciones en las decisiones que toman los pescadores en la asignación del esfuerzo de pesca. Por ejemplo, Moreno-Báez et al. (2010) validaron información sobre la distribución espacial del esfuerzo mediante la incorporación del conocimiento local de pescadores usando un proceso participativo de talleres y entrevistas. Específicamente, el conocimiento captado por MorenoBáez y colaboradores (2010) responde al concepto de mapas mentales, que representa el conocimiento individual de un área a través de la percepción y la memoria. Estos mapas mentales se generan a partir de la percepción de características biológicas y sociales en el ambiente marino y la forma como estas características se interrelacionan.

Conceptualmente ligada al PC, la teoría del bienestar ha sido mencionada en estudios sociales aplicados a comunidades pesqueras (Weeratunge et al. 2013). Este concepto provee una amplia visión social del beneficio derivado de la actividad pesquera, que trasciende la visión comúnmente tomada en cuenta en marcos pesqueros donde imperan componentes económicos. Según Weeratunge et al. (2013), el enfoque del bienestar engloba una amplia base de resultados, que van desde metas materiales, como producción económica, soporte alimenticio y empleo, hasta aspectos no materiales, como seguridad, condi- 
ciones de trabajo no discriminatorias o preservación de valores ecológicos de los sistemas marinos y satisfacción laboral. Estos últimos aspectos subjetivos han sido descritos y cuantificados por varios autores que han abordado el tema de satisfacción laboral. Por ejemplo, según Spector (1997) la satisfacción por el trabajo se origina desde dos ámbitos: i) factores individuales que investigan la relación entre los aspectos personales y la satisfacción laboral; ii) el factor relacionado con la naturaleza del trabajo y el ambiente, y se enfoca en la investigación de la percepción de los individuos con respecto al trabajo. Pollnac \& Poggie (2008) argumentan que los pescadores presentan una dimensión de satisfacción laboral diferente a los trabajadores de otros sectores productivos, debido a factores como: la emoción de la caza, el desafío de enfrentar el poder y la extensión del mar, y la aventura global de enfrentarse a uno mismo contra los elementos y la búsqueda de pescado; lo que agrega un componente de autorrealización personal que incluye la "aventura" y el "desafío". Los autores señalan que la naturaleza relativamente arriesgada de la ocupación de la pesca atrae y retiene a los individuos caracterizados por ser activos, de aventura, agresivos y de personalidad valiente, por lo que estos componentes de riesgo laboral tienen una influencia positiva en sus niveles de felicidad.

Los aspectos antes mencionados influyen directamente en el proceso de decisiones de los pescadores en el corto y largo plazos, aunque pocas técnicas de modelación logran incorporar estos aspectos en el análisis de la conducta del pescador. Como indican Pollnac \& Poggie (2006), "la combinación de características personales como actitudes atrevidas, agresivas, y aventureras, factores culturales (por ejemplo, creencias religiosas), genéticos y uso de drogas y alcohol reducen la percepción del riesgo entre pescadores", lo que podría, por un lado, promover los accidentes durante las faenas operacionales (por ejemplo, descompresión en buceo artesanal) y, por el otro, influir en la eficiencia y esfuerzo de pesca (Naranjo-Madrigal, 2014). Además, los mismos autores (Pollnac \& Poggie, 2008) señalan que la satisfacción laboral y la felicidad, derivada de la actividad pesquera, son muy importantes para entender el grado de resistencia de los pescadores a cambios en la pesquería (por ejemplo, disminución del recurso), lo que puede ejercer presión más allá del máximo rendimiento económico y promover la sobreexplotación de los recursos.

Una de las causas que explica el fuerte apego al trabajo que presentan los pescadores fue explorada en un estudio realizado con pescadores de pequeña escala en Uruguay y Brasil (Trimble \& Johnson, 2013). En este estudio, los autores demostraron que los pescadores consideran sus actividades en este sector productivo como un estilo de vida más que un trabajo, esto porque en sus motivaciones ocupacionales existen aspectos placenteros tanto monetarios como no monetarios que los hacen 
mantenerse en la actividad. Otro factor que se relaciona con el apego al trabajo es la condición de pesca de subsistencia que presentan las pesquerías artesanales mixtas, donde aspectos económicos y de seguridad alimentaria se combinan. En estos casos, la pesca representa una estrategia de sobreviviencia y es de importancia en la economía local (Busilacchi et al. 2013). Por ejemplo, Kronen (2004) reportó en Tonga que una proporción de la captura era consumida por los pescadores o compartida con sus allegados y no entraba en el mercado. La captura que entraba en el mercado era un resultado de la necesidad de dinero en efectivo y no una respuesta a la demanda del mercado.

No hay duda que pesquerías de pequeña escala en países en vías de desarrollo están asociadas a comunidades cuyos pobladores enfrentan condiciones de vida extremadamente graves, muy diferentes a las condiciones socioeconómicas de comunidades de zonas urbanas en países desarrollados. Por ejemplo, Béné (2009), en un estudio sobre pescadores de pequeña escala en el Congo, señaló que las condiciones de falta de desarrollo, marginación y aislamiento económico no eran exclusivas de la comunidad de pescadores, también afectaban a otros grupos socioeconómicos de la región. Además, este autor pudo identificar pescadores eficientes, cuyo promedio de ingresos era más alto que en otros hogares encuestados.

Existen otras aproximaciones que han sido utilizadas para entender aspectos socioculturales y económicos de las comunidades asociadas a la pesca artesanal, con el fin de incluir estos aspectos en objetivos de manejo (por ejemplo, Béné, 2009; Cinner et al. 2012). Sin embargo, queda claro que las respuestas a las preguntas que se plantean los pescadores en su diario vivir: ¿cómo?, ¿cuándo?, ¿dónde? y ¿qué pescar? no pueden ser respondidas de forma amplia a menos que se rastreen a profundidad las motivaciones detrás del proceso de toma de decisiones de los pescadores dados diferentes contextos. Otras preguntas como: ¿por qué trabajan en la pesca?, ¿por qué les gusta pescar?, ¿cuáles son las futuras aspiraciones para usted y sus hijos? (Trimble \& Johnson, 2013) ¿cambiarían la pesca por otra forma de trabajo?, ayudan a esclarecer complejos aspectos del estilo de vida de los pescadores que influyen en su conducta.

\section{DISCUSIÓN}

En esta revisión de literatura se confirma la gran asimetría existente en la inclusión de motivadores de la conducta del pescador en casos de estudio, por ejemplo, el contraste entre los estudios sociales con respecto a los estudios económicos. Asimismo, se observó que algunas técnicas de modelación fueron más robustas que otras al considerar una amplia gama de variables cualitativas y cuantitativas implícitas en la toma de decisiones del pescador (por ejemplo, modelos de redes en contraste con modelos individuales).

Implicaciones en los procesos de gestión de pesquerías

Esta revisión de literatura ha permitido sintetizar diversas aproxima- 
ciones teóricas y técnicas de modelación analítica que ha conllevado el estudio de múltiples aspectos que son claves para comprender la conducta de los pescadores de pequeña escala, así como las decisiones que toman durante las operaciones de pesca, su reacción ante regulaciones, variación en la disponibilidad del recurso y eventos ambientales, con el fin de desarrollar planes de gestión eficaces (por ejemplo, Béné \& Tewfik, 2001; Salas \& Gaertner, 2004). No obstante, como se constató en esta revisión, la conducta del pescador es contexto-dependiente, lo que quiere decir que está supeditada a la gran heterogeneidad y dinamismo existente en las comunidades pesqueras definidas por aspectos geográficos, socioculturales y ambientales (Fabinyi et al. 2013; Reed et al. 2013). Esto hace que los pescadores tengan diferentes respuestas ante medidas de manejo y que su forma de interacción con el entorno sea compleja.

En los casos de estudio analizados, se hace énfasis a la incorporación del conocimiento de aspectos de la conducta de los pescadores en procesos de gestión, lo que obliga a construir capacidades de manejo para adaptar a las políticas, las formas de operación, estilo de vida y situación socioeconómica de los pescadores. Sistemas de manejo que se ajustan al proceso de incorporación de estos aspectos han sido propuestos en la literatura, y existen ejemplos en muchas pesquerías de pequeña escala alrededor del mundo (Ahmed et al. 2013; Plagányi et al. 2013). Se destacan el comanejo (Pomeroy \& Berkes, 1997), el manejo adaptativo (Plummer \& Armitage, 2007), el manejo basado en la comunidad (King \& Faasili, 2001) $\mathrm{y}$ el enfoque ecosistémico (FAO, 2003). Estos sistemas de manejo se caracterizan por ser participativos, integrando a la vez componentes del recurso y socioeconómicos. Fulton et al. (2011) proponen el manejo integrado, el cual permite incluir una variedad de herramientas políticas que son más consistentes a la incertidumbre del comportamiento e implementación, ya que combinan apoyos de gestión complementarios que conducen a una convergencia en los incentivos y los objetivos de gestión. Este sistema de manejo tiene esta capacidad, dado que utiliza la comprensión de la relación entre las motivaciones de comportamiento específicas y las diferentes herramientas de gestión (Fulton et al. 2011), para optimizar la combinación de políticas para un sistema en particular.

\section{Modelo conceptual de manejo socioecológico}

Los estudios abordados en esta revisión demuestran la necesidad de ampliar las herramientas de gestión tradicional de pesquerías hacia herramientas que integren la complejidad e incertidumbre inherentes a los múltiples motivadores de la conducta del pescador y su relación con el componente biofísico (recursos y medio ambiente) del cual depende y es parte. Con este fin y como resultado de la revisión de sólidos estudios de caso, se propone un modelo conceptual de manejo basado en los 
sistemas socioecológicos (adaptado de Ommer et al. 2012), dada la robustez de esta aproximación al analizar la interacción entre el ser humano y la naturaleza, los derechos, las reglas y los valores de la colectividad, integrar a las organizaciones comunales, su carácter multidimensional y flexibilidad en el manejo (Fig. 2). El término de sistema socioecológico es un concepto holístico, sistémico e integrador del "ser humano-en-la naturaleza". Por lo tanto, se entiende como un sistema complejo y adaptativo, en el que distintos componentes culturales, políticos, sociales, económicos, ecológicos y tecnológicos están interactuando (Resilience Alliance, 2010). Esto implica que el enfoque de la gestión de los ecosistemas y recursos naturales no se centra en los componentes de la palabra sistema sino en sus relaciones, interacciones y retroalimentaciones. El estudio de un sistema socioecológico se puede abordar analizando los mecanismos que llevan a la sostenibilidad de un sistema.

$\mathrm{Al}$ igual que el manejo integrado, el modelo conceptual de manejo propuesto en este estudio incorpora factores externos de cambio representados por tendencias, eventos o políticas que afectan la conducta humana y los procesos ecológicos locales (Kittinger et al. 2013). Este modelo conceptual de manejo socioecológico debe incorporar el enfoque adaptativo, el cual responde al dinamismo y a la complejidad que muestra la conducta del pescador, ya que enfrenta el desafío del cambio constante en los sistemas y permite el aprendizaje progresivo no solo individual, sino también comunitario, institucional, político y ecológico (Plummer \& Armitage, 2007). En contraste al modelo bioeconómico tradicional de manejo, que da peso a modelos individuales de especies comerciales exclusivamente, programas que limitan el acceso a los recursos, la perspectiva de ver a los pescadores como individuos económicamente racionales cuya conducta puede ser predicha, controlada y manipulada a través de iniciativas de manejo (Ommer et al. 2012), el modelo conceptual de manejo planteado (Fig. 2) es una alternativa holística que se destaca por la justicia social, dado que capta aspectos sensibles de las comunidades mediante las aproximaciones diagnóstico (supervivencia, bienestar, vulnerabilidad, servicios ecosistémicos, resiliencia) y, además, las interacciones del ser humano con los recursos (Kittinger et al. 2013). Según Ommer et al. (2012), la importancia de atender el impacto del cambio climático y la globalización sobre las actividades humanas mediante diferentes fuentes de conocimiento de los ecosistemas marinos es crucial, pero su análisis requiere que se considere a las sociedades humanas como parte integrante de los sistemas marinos y que los procesos de resolución de problemas socioambientales estén orientados por aproximaciones de investigación inter y transdisciplinarios (Lang et al. 2012).

Debido a lo anterior, al presente modelo conceptual de manejo socioecológico se le han adaptado conceptos recientes que han surgido a 


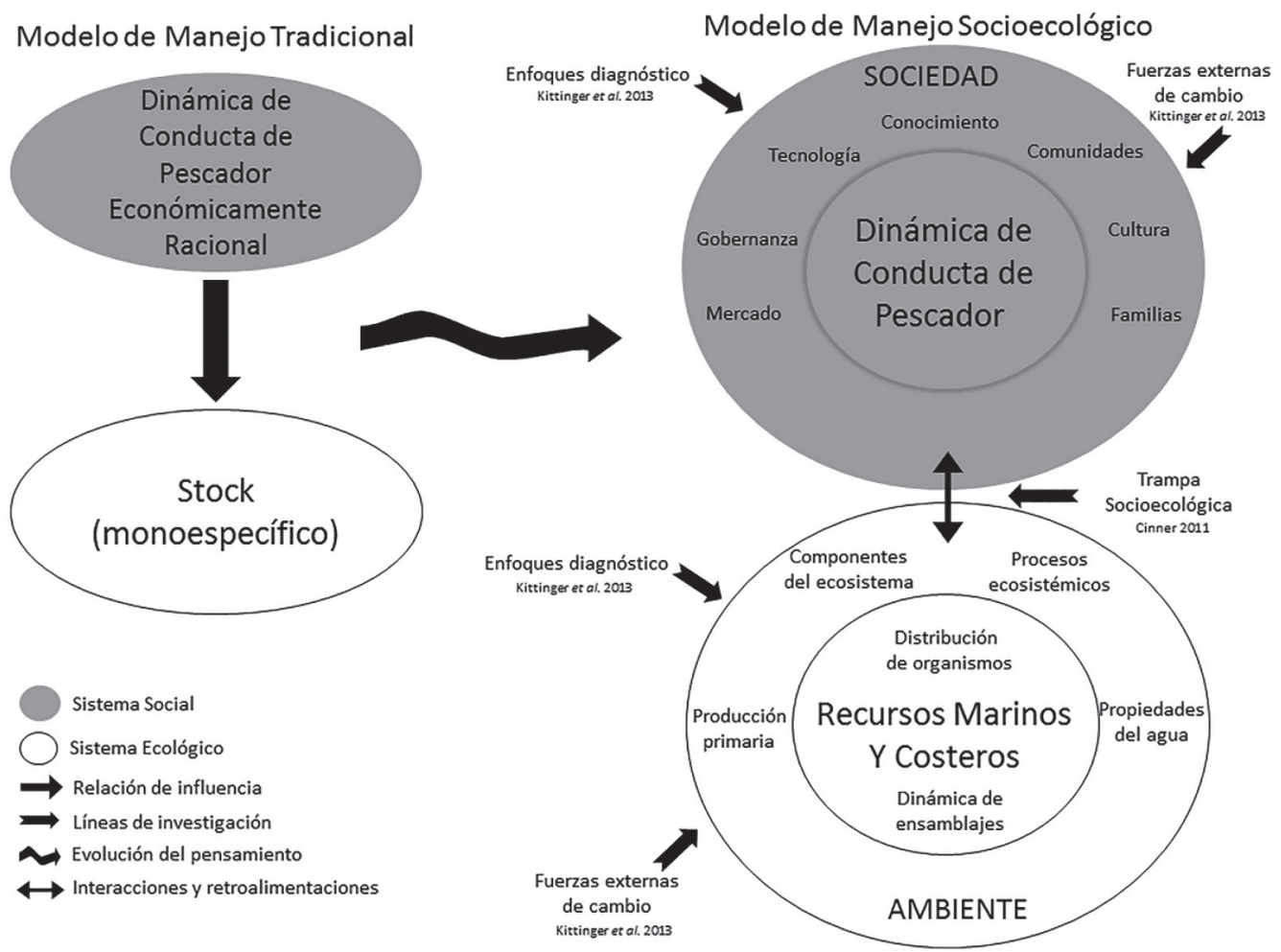

Fig. 2. Evolución del modelo de manejo pesquero tradicional al modelo de manejo socioecológico. Adaptado de Ommer et al. 2012

Fig. 2. Evolution of the traditional fishery management framework to the socioecological fishery management framework. Adapted from Ommer et al. 2012

partir de estudios sociales y estudios sobre gobernanza de sistemas socioecológicos aplicados a pesquerías de pequeña escala (Kittinger et al.2013). Estos conceptos integran aspectos específicos que han sido abordados en estudios de la conducta del pescador. A la derecha de la figura 2, se observa la evolución al modelo conceptual de manejo socioecológico que está compuesto por un círculo concéntrico que simboliza la dinámica de la conducta del pescador, la cual puede ser influida por muchos factores que son parte del sistema social del cual es componente, desde aspectos tecnológicos hasta sistemas de conocimiento, en contraste al modelo reduccionista tradicional de manejo (situado a la izquierda de la figura 2), basado en aspectos económicos, paradigmas mercantilistas $\mathrm{y}$ en un pescador económicamente racional. El sistema social (coloración gris) del modelo conceptual de manejo socioecológico es interdependiente mediante interacciones y retroalimentaciones (flecha negra bidireccional) que se establecen con el sistema ecológico (círculos blancos en la parte inferior), el cual engloba los 
recursos marino-costeros representados por las especies de interés comercial y el ecosistema del cual son parte, esta relación se basa en la literatura asociada a la aproximación de ecosistemas (Rice, 2011). Las interdependencias de estos sistemas son dinámicas $\mathrm{y}$ cambiantes. Por ejemplo, motivaciones socioculturales, económicas y legales afectan la conducta del pescador y, por ende, la actividad pesquera de la comunidad y esto, a su vez, afectará los ensambles de peces y el ecosistema marino con el que interactúan (Ommer et al. 2012). Por lo tanto, este modelo conceptual de manejo captura el proceso de toma de decisiones de los pescadores en su rango más amplio, que se extiende a partir de aspectos del contexto comunitario.

Adicionalmente, se integran a este modelo conceptual de manejo, las líneas de investigación planteadas por Kittinger et al. (2013), que ayudarían a mejorar la sostenibilidad de los sistemas socioecológicos, haciendo especial énfasis en la inclusión de motivadores antropogénicos: i) las fuerzas externas de cambio representadas por tendencias, eventos y políticas que afectan la conducta humana y los procesos ecológicos locales en los sistemas socioecológicos; ii) trampas socioecológicas definidas por "respuestas (instituciones ausentes o débiles, trampas de pobreza, economía en depresión, artes de pesca destructivos) entre los sistemas sociales y ecológicos que llevan hacia un estado indeseable, que puede ser difícil o imposible revertir" (Cinner, 2011); iii) aproximaciones de diagnóstico que permiten caracterizar atributos de los sistemas socioecológicos y que integran los enfoques sociales con los cuales se ha estudiado la conducta del pescador. Estos enfoques reconocen que las relaciones causa y efecto simplistas siempre fallan a la hora de explicar la complejidad de las relaciones socioecológicas.

Además, existen prerrequisitos políticos que, con base en el modelo conceptual de manejo propuesto, ayudarían a mejorar la sostenibilidad de los sistemas socioecológicos como: áreas protegidas, restricciones de artes de pesca, incentivos económicos y aproximaciones basadas en derechos de propiedad (Kittinger et al. 2013). Sin embargo, todavía es necesario que se desarrollen las capacidades de manejo basadas en procesos participativos, principalmente en los países en vías de desarrollo, que auxilien en el diseño e implementación de medidas fundamentadas en modelos conceptuales de manejo integrales como el propuesto en este estudio y el enfoque de gobernanza adaptativa (ver Österblom et al. 2013). Este es el reto que los gobiernos, las organizaciones no gubernamentales, los científicos pesqueros y sociales deben enfrentar, con esfuerzos conjuntos para lograr un adecuado entendimiento y manejo de los sistemas pesqueros.

\section{CONCLUSIONES}

Las teorías y los modelos que han analizado la conducta del pescador son variados y presentan una gran asimetría 
en la diversidad de motivadores que logran explicar y predecir el comportamiento. Sin embargo, los modelos de redes parecen ser los más robustos al tener la capacidad de incorporar gran cantidad de motivadores de distinta índole (por ejemplo, monetarios y no monetarios) y que están implícitos en las elecciones que hacen los pescadores en el corto y largo plazos.

Por lo tanto, enfoques de investigación inter y transdisciplinarios deben impulsar los análisis dirigidos al estudio de la conducta del pescador, con el fin de explicar mejor sus decisiones asociadas al esfuerzo, incentivos económicos, capturas, regulaciones, aspectos de su cultura, forma de vida, estrategias de sobrevivencia, satisfacción laboral y percepción del bienestar. Lo anterior debe complementarse con procesos de gestión participativos y holísticos que ayuden a incorporar este conocimiento en los objetivos de manejo.

El modelo conceptual socioecológico de manejo propuesto puede ser usado como una hoja de ruta para la construcción de herramientas de modelación (por ejemplo, modelos de redes), con el fin de evaluar potenciales interacciones y resultados entre las motivaciones que influyen en las acciones del pescador (por ejemplo, incentivos económicos, regulaciones) y su efecto sobre el uso de especies de interés comercial, así como el impacto de este aprovechamiento sobre otros factores ecosistémicos (por ejemplo, redes tróficas y factores biogeoquímicos).

\section{AGRADECIMIENTOS}

El autor desea agradecer a los revisores anónimos por sus valiosas sugerencias.

\section{BIBLIOGRAFÍA}

Abernethy, K., Allison, E. H., Molloy, P. \& Cote, I. (2007). Why do fishers fish where they fish? Using the ideal free distribution to understand the behaviour of artisanal reef fishers. Can. J. Fish. Aqua. Sci., 64, 1595-1604. http:/á/dx.doi.org/10.1139/f07-125

Ahmed, N., Rahman, S., Bunting, S. \& Brugere, C. (2013). Socio-economic and ecological challenges of smallscale fishing and strategies for its sustainable management: A case of study of the Old Brahmaputra River, Bangladesh. Singap. J. Trop. Geogr., 34, 86-102. http://dx.doi.org/10.1111/ sjtg. 12015

Al-Subhi, K., Bose, S. \& Al-Masroori, S. (2013). Fishers' compliance motivations: A case study of the Sultanate of Oman. Mar. Pol., 37, 141148. http://dx.doi.org/10.1016/j. marpol.2012.03.015

Álvarez, P., Espejel, I. \& Bocco, G. (2012). Comunidades pesqueras y paisaje cultural: una revisión de los enfoques de investigación. Costas, 1, 10-26.

Anderson, R. \& Carter, F. (1990). Human Behavior in the Social Environment: A Social Systemic Approach. New York, EE. UU.: Walter de Gruyter Inc.

Béné, C. \& Tewfik, A. (2001). Fishing effort allocation and fishermen's decision making process in a multi-species small-scale fishery: analysis of the conch and lobster fishery in Turks and Caicos Islands. Hum. Ecol., 29, 157-186. http://dx.doi. org/10.1023/A:1011059830170 
Béné, C. (2009). Are fishers poor or vulnerable? Assessing economic vulnerability in small-scale fishing communities. J. Dev. Stud., 45, 911-933. http://dx.doi. org/10.1080/00220380902807395

Berkes, F. (1999). Sacred ecology. Traditional ecological knowledge and resource management. Philadelphia, EE. UU.: Taylor \& Francis.

Bockstael, N. \& Opaluch, J. (1983). Discrete Modelling of Supply Response under Uncertainty: The Case of the Fishery. J. Environ. Econ. Manage., 10(2), 125-137. http://dx.doi. org/10.1016/0095-0696(83)90021-9

Busilacchi, S., Russ, G., Williams, A., Sutton, S. \& Begg, G. (2013). The role of subsistence fishing in the hybrid economy of an indigenous community. Mar. Pol., 37, 183-191. http://dx.doi. org/10.1016/j.marpol.2012.04.017

Butler, R., Tawake, A., Skewes, T., Tawake, L. \& McGrath, V. (2012). Integrating traditional ecological knowledge and fisheries management in the Torres Strait, Australia: the catalytic role of turtles and dugong as cultural keystone species. Ecol. Soc., 17(4), 34. http://dx.doi.org/10.5751/ ES-05165-170434

Cabrera, J. \& Defeo, O. (2001). Daily bioeconomic analysis in a multispecific artisanal fishery in Yucatan, Mexico. Aquat. Living Res., 14, 19-24. http://dx.doi.org/10.1016/ S0990-7440(00)01094-9

Cinner, J. (2011). Social-ecological traps in reef fisheries. Glob. Environ. Chang., 21, 835-839. http://dx.doi. org/10.1016/j.gloenvcha.2011.04.012

Cinner, J. E., McClanahan, T. R., Graham, N. A. J., Daw, T. M., Maina, J., Stead, S. M., Wamukota, A., Brown, K. \& Bodin, O. (2012). Vulnerability of coastal communities to key impacts of climate change on coral reef fisheries. Glob. Environ. Chang., 22, 12-20. http://dx.doi.org/10.1016/j. gloenvcha.2011.09.018

Davidson-Hunt, I. (2003). Indigenous lands management, cultural landscapes and Anishinaabe people of Shoal Lake, Northwestern Ontario, Canada. Environ., 31, 21-41.

Dietrich, C. (2010). Decision Making: Factors that Influence Decision Making, Heuristics Used, and Decision Outcomes. Student Pulse, 2(02), 1-3.

Dreyfus-León, M. (1999). Individual-based modelling of fishermen search behaviour with neural networks and reinforcement learning. Ecol. Model., 120, 287-297. http://dx.doi. org/10.1016/S0304-3800(99)00109-X

Fabinyi, M., Foale, S. \& Macintyre, M. (2013). Managing inequality or managing stocks? An ethnographic perspective on the governance of small-scale fisheries. Fish. Fish., 16(3), 471-485. http://dx.doi.org/10.1111/faf.12069

FAO. (2003). The Ecosystem Approach to Fisheries. FAO Technical Guidelines for Responsible Fisheries. 4, Suppl. 2. Rome: Publishing Management Service, Information Division.

FAO. (2012). The State of World Fisheries and Aquaculture. Rome: Publishing Management Service, Information Division.

Fretwell, S. (1972). Populations in a seasonal environment. Monogr. Рори. Biol., 91(1), 204-207.

Fukuda, D. \& Yai, T. (2010). Semiparametric Specification of the Utility Function in a Travel Mode Choice Model. Transportation, 37(2), 221-238. http://dx.doi.org/10.1007/ s11116-009-9253-5

Fulton, E., Smith, A., Smith, D. \& van Putten, I. (2011). Human 
behaviour: the key source of uncertainty in fisheries management. Fish. Fish., 12, 2-17. http://dx.doi. org/10.1111/j.1467-2979.2010.00371.x Gordon, S. (1953). An economic approach to the optimum utilization of fishery resources. J. Fish. Res. Board. Can., 10(7), 442-457. http://dx.doi. org/10.1139/f53-026

Handel, M. (1989). War, Strategy, and Intelligence. New York, EE. UU.: Routledge.

Hilborn, R. (2007). Moving to sustainability by learning from successful fisheries. Ambio., 36, 296-303. http://dx.doi. org/10.1579/0044-7447(2007)36[296:MT SBLF]2.0.CO;2

Holland, D. \& Sutinen, J. (1999). An empirical model of fleet dynamics in New England trawl fisheries. Can. J. Fish. Aquat. Sci., 56, 253-264. http://dx.doi. org/10.1139/f98-169

Holland, D. (2008). Are fishermen rational? A fishing expedition. Mar. Resour. Econ., 23, 325-344. http://dx.doi. org/10.1086/mre.23.3.42629621

King, M. \& Faasili, U. (2001). Community-based management of subsistence fisheries in Samoa. Fish. Manag. Ecol., 6, 133-144. http://dx.doi.org/10.1046/ j.1365-2400.1999.00136.x

Kittinger, J., Finkbeiner, E., Ban, N., Broad, K., Carr, M., Cinner, J., Gelcich, S., Cornwell, M., Koehn, J., Basurto, X., Fujita, R., Caldwell, R. \& Crowder, L. (2013). Emerging frontiers in social-ecological systems research for sustainability of small-scale fisheries. Curr. Opin. Environ. Sustain., 5(3), 352-357. http:// dx.doi.org/10.1016/j.cosust.2013.06.008

Kolaczyk, E. \& Csárdi, G. (2014). Statistical Analysis of Network Data with $R$. Springer Science+Business Media. New York, EE. UU.: Springer.
Kronen, M. (2004). Fishing for fortunes? A socio-economic assessment of Tonga's artisanal fisheries. Fish. Res., 70, 121-134. http://dx.doi.org/10.1016/ s0165-7836(04)00161-4

Kuperan, K. \& Sutinen, J. (1998). Blue water crime: deterrence, legitimacy, and compliance in fisheries. Law Soc. Rev., 32, 309-337. http://dx.doi. org/10.2307/827765

Lang, J., Wiek, A., Bergmann, M., Stauffacher, M., Martens, P., Moll, P., Swilling, M. \& Thomas, C. J. (2012). Transdisciplinary research in sustainability science: practice, principles, and challenges. Sustain. Scie., 7(S1), 25-43. http://dx.doi.org/10.1007/ s11625-011-0149-x

Mas-Colell, A., Whinston, M. \& Green, J. (1995). Microeconomic Theory. New York, EE. UU.: Oxford.

May, P. (2004). Compliance motivations: affirmative and negative bases. Law. Soc. Rev., 38, 41-68. http://dx.doi.org/10.1111/ j.0023-9216.2004.03801002.x

Moreno-Báez, M., Orr, B., CudneyBueno, R. \& Shaw, W. (2010). Using fishers' local knowledge to aid management at regional scales: spatial distribution of small-scale fisheries in the northern Gulf of California, Mexico. B. Mar. Sci., 86, 339-353.

Naranjo-Madrigal, H. (2014). Análisis espacio-temporal de las operaciones de pesca de las pesquerías artesanales bento-demersales en Playa Lagarto, Costa Rica. Tesis de doctorado no publicada, Centro de Estudios Avanzados del IPN, CINVESTAV, México.

Naranjo-Madrigal, H., van Putten, I. \& Norman-López, A. (2015). Understanding socio-ecological drivers of spatial allocation choice in a multispecies artisanal 
fishery: a Bayesian network modeling approach. Mar. Pol. 62, 102-115. http:// dx.doi.org/10.1016/j.marpol.2015.09.003 Nøstbakken, L. (2008). Fisheries law enforcement - a survey of the economic literature. Mar. Pol., 32, 293300. http://dx.doi.org/10.1016/j. marpol.2007.06.002

Ommer, R., Perry, R., Murray, G. \& Neis, B. (2012). Social-ecological dynamism, knowledge, and sustainable coastal marine fisheries. Curr. Opin. Environ. Sustain., 4(3), 316-322. http://dx.doi. org/10.1016/j.cosust.2012.05.010

Österblom, H., Merrie, A., Metian, M., Boonstra, W., Blenckner, T., Watson, J., Rykaczewski, R., Ota, Y., Sarmiento, J., Christensen, V., Schluter, M., Birnbaum, S., Gustafsson, G., Humborg, C., Mörth, C., Múller-Karulis, B., Tomczak, M., Troell, M. \& Folke, C. (2013). Modelling social-ecological scenarios in marine systems. BioScience, 63, 735-744. http://dx.doi.org/10.1093/ bioscience/63.9.735

Ota, Y. \& Just, R. (2008). Fleet sizes, fishing effort and the 'hidden' factors behind statistics: an anthropological study of small-scale fisheries in UK. Mar. Pol., 32, 301-308. http://dx.doi. org/10.1016/j.marpol.2007.06.006

Pearl, J. (1998). Probabilistic Reasoning in Intelligent Systems: Networks of Plausible Inference. Palo Alto, EE. UU.: Morgan Kaufmann Publishers.

Pitcher, T. \& Cheung, W. (2013). Fisheries: hope or despair? Mar. Pollut. Bull., 74, 506-516. http://dx.doi. org/10.1016/j.marpolbul.2013.05.045

Plagányi, E., van Putten, I., Hutton, T., Deg, A., Dennis, D., Pascoe, S., Skewes, T. \& Campbell, A. (2013). Integrating indigenous livelihood and lifestyle objectives in managing a natural resource. PNAS, 110, 3639-3644. http:// dx.doi.org/10.1073/pnas.1217822110

Plummer, R. \& Armitage, D. (2007). A resilience-based framework for evaluating adaptive comanagement: linking ecology, economics and society in a complex world. Ecol. Eco., 61, 62-74. http://dx.doi.org/10.1016/j. ecolecon.2006.09.025

Pollnac, R. \& Poggie, J. (2006). Job satisfaction in the fishery in two Southeast Alaskan towns. Hum. Organ., 6, 329-339. http://dx.doi.org/10.17730/ humo.65.3.3j2w39a21tq3j411

Pollnac, R. \& Poggie, J. (2008). Happiness, well-being and psychocultural adaptation to the stresses associated with marine fishing. Hum. Eco. Rev., 15, 194-200.

Pomeroy, R. \& Berkes, F. (1997). Two to tango: the role of government in fisheries co-management. Mar. Pol., 21,465-480. http://dx.doi.org/10.1016/ S0308-597X(97)00017-1

Prato, T. (2005). Bayesian adaptive management of ecosystems. Ecol. Model., 183, 147-156. http://dx.doi. org/10.1016/j.ecolmodel.2004.07.024

Railsback, S. (2001). Concepts from complex adaptive systems as a framework for individual-based modelling. Ecol. Model., 139, 47-62. http://dx.doi. org/10.1016/S0304-3800(01)00228-9

Reed, M., Courtney, P., Urquhart, J. \& Ross, N. (2013). Beyond fish as commodities: Understanding the socio-cultural role of inshore fisheries in England. Mar. Pol., 37, 62-68. http://dx.doi. org/10.1016/j.marpol.2012.04.009

Resilience Alliance. (2010). Assessing resilience in social-ecological systems: workbook for practitioners. Recuperado en abril 14, 2016, disponible en: 
http://www.resalliance.org/index.php/ resilience assessment.

Rice, J. (2011). Managing fisheries well: delivering the promises of an ecosystem approach. Fish. Fish., 12, 209-231. http://dx.doi. org/10.1111/j.1467-2979.2011.00416.x

Salas, S. \& Gaertner, D. (2004). The behavioural dynamics of fishers: Management implications. Fish. Fish., 5, 153-167. http://dx.doi. org/10.1111/j.1467-2979.2004.00146.x

Salas, S., Sumaila, U. \& Pitcher, T. (2004). Short-term decisions of small-scale fishers selecting alternative target species: a choice model. Can. J. Fish. Aquat. Sci., 61, 374383. http://dx.doi.org/10.1139/f04-007

Spector, P. (1997). Job satisfaction. Application, assessment, causes, and consequences. Thousand Oaks, CA, EE. UU.: Sage Publications.

Stanovich, K. \& West, R. (2008). On the relative independence of thinking biases and cognitive ability. J. Pers. Soc. Psychol., 94(4), 672-695. http://dx.doi. org/10.1037/0022-3514.94.4.672

Stevens, K., Frank, K. \& Kramer, D. (2015). Do Social Networks Influence Small-Scale Fishermen's Enforcement of Sea Tenure? PLoSONE, 10(3), e0121431. http://dx.doi. org/10.1371/journal.pone.0121431

Stevenson, T., Brian, N. \& Dierking, J. (2011). Fisher behaviour influences catch productivity and selectivity in West Hawaii's aquarium fishery. ICES.
J. Mar. Sci., 68, 813-822. http://dx.doi. org/10.1093/icesjms/fsr020

Trimble, M. \& Johnson, D. (2013). Artisanal fishing as an undesirable way of life? The implications for governance of fishers' wellbeing aspirations in coastal Uruguay and southeastern Brazil. Mar. Pol., 37, 37-44. http://dx.doi. org/10.1016/j.marpol.2012.04.002

van Putten, I., Kumala, S., Thébaud, O., Dowling, N., Hamon, K. G., Hutton, T. \& Pascoe, S. (2012). Theories and behavioural drivers underlying fleet dynamics models. Fish. Fish., 13, 216-235. http://dx.doi. org/10.1111/j.1467-2979.2011.00430.x van Putten, I., Lalancette, A., Bayliss, P., Dennis, D., Hutton, T., NormanLópez, A., Pascoe, S., Plagányi, E. \& Skewes, T. (2013). A Bayesian model of factors influencing indigenous participation in the Torres Strait tropical rock lobster fishery. Mar. Pol., 37, 96-105. http://dx.doi.org/10.1016/j. marpol.2012.04.001

Weeratunge, N., Béné, C., Siriwardane, R., Charles, A., Johnson, D., Allison, E., Nayak, P. K. \& Badjeck, M. (2013). Small-scale fisheries through the wellbeing lens. Fish. Fish., 15(2), 255-279. http://dx.doi.org/10.1111/faf.12016

Worm, B. \& Branch, T. (2012). The future of fish. Trend Ecol. Evol., 27, 594-599. http://dx.doi.org/10.1016/j. tree.2012.07.005 